\title{
Analytical and Numerical Solutions of Contact Problem for the Microperiodic Slant Layered Composite Body in A Plane Strain State
}

\author{
Piotr Sebestianiuk ${ }^{1}$, Pawel Mościcki ${ }^{1}$, Dariusz Perkowski ${ }^{1}$, \\ ${ }^{1}$ Faculty of Mechanical Engineering/Bialystok University of Technology \\ Wiejska 45C street, 15-351 Białystok, Poland \\ p.sebestianiuk@doktoranci.pb.edu.pl; pawel.moscicki.96@gmail.com \\ d.perkowski@pb.edu.pl;
}

\section{Extended Abstract}

Contact problems of elastic solids are widely studied due to their engineering applications when considering contact of friction pairs or other processes related to the transfer of load from one body to another for both homogeneous and heterogeneous materials - including materials with structural gradation of properties or composites. For this type of materials, solving problems and describing mechanical properties often comes down to the use of various averaging methods, e.g. homogenization methods. One of the examples of such methods is the homogenization method with microlocal parameters used, among others, for heterogeneous composite materials.

Author's main scope of research are composite materials with layered structure - especially microperiodic layered composites [1-4]. For this type of materials, solutions for layers perpendicular and parallel to the edge are widely known, and the inclined layering is still under investigation. This work presents a plane contact problem for the microperiodic composite half space with slant layering. Presented work discuss a contact of a rigid punch pressed into the composite body with normal forces in the state of limit equilibrium. Two punch shapes were considered - cylindrical and rectangular. Microperiodic composite under consideration is made from thin layers arranged periodically. Each layer is made of two homogenous components differing in their Young's modules.

Presented problem has been solved analytically using the homogenized model with microlocal parameters and numerically using a FEM. Analytical results in the form of the contact pressures and stress distributions are presented for both components of the composite body in the integral form. The integrals have been computed numerically. Characteristics of maximum pressures and width of the contact zone are presented for different angles of stratification and for different stiffness ratios of composite components. The results are presented for both punch shapes. Authors are testing the applicability of the homogenized model based on FEM solutions, because analytical solutions under the classical theory of elasticity are almost impossible to obtain, and the time spent on it is too long.

In the case of the per-component contact pressures it could be seen that their maximum values differ the more the higher stiffness ratios are, so considering only the averaged values is not justified and an approach that allows to determine the stresses in each layer separately should be used - as is possible with the homogenized model with microlocal parameters.

\section{Acknowledgements}

The research has been conducted as part of projects at the Faculty of Mechanical Engineering of the Bialystok University of Technology. Project number WZ/WM-IIM/3/2020.

\section{References}

[1] P. Sebestianiuk, D. M. Perkowski, R. Kulchytsky-Zhyhailo, "On contact problem for the microperiodic composite half-plane with slant layering," Int. J. Mechanical Sci., vol. 182 , pp.1-16, 2020.

[2] C. Woźniak, "A nonstandard method of modelling of thermoelastic periodic composites," Int. J. Eng. Sci., vol. 25, no. 4, pp. 483-498, 1987.

[3] R. Kulchytsky-Zhyhailo, S. J. Matysiak, "On three-dimensional problems in a semi-infinite periodically layered body," J. Theoretical an Applied Mech., vol. 33, pp. 771-781, 1995.

[4] D. M. Perkowski, S. J. Matysiak, „On heat conduction in a semi-infinite laminated layer. Comparative results for two approaches", Int. Comm. Heat and Mass Transfer", vol. 37, no.4, pp. 343-349, 2010. 